\title{
COUNTING SUBGROUPS OF NON-EUCLIDEAN CRYSTALLOGRAPHIC GROUPS
}

\author{
GARETH A. JONES
}

\begin{abstract}
A method is obtained for counting the normal subgroups $N$ of a noneuclidean crystallographic group $\Gamma$ without reflections, with a given finite quotient group $\Gamma / N$; this has applications to the enumeration of regular coverings of orbifolds. The method, which involves Möbius inversion and character theory, is also applied to count normal surface subgroups and non-normal subgroups of finite index in $\Gamma$.
\end{abstract}

\section{Introduction}

The aim of this note is to describe and to illustrate a technique for computing the number $n_{\Gamma}(G)$ of normal subgroups $N$ of a non-euclidean crystallographic group (or NEC group) $\Gamma$, with quotient group $\Gamma / N$ isomorphic to a given finite group $G$. The first part of the method (due to P. Hall) uses Möbius inversion to reduce the problem to that of counting homomorphisms from $\Gamma$ to various subgroups of $G$; this can be applied to any finitely generated group $\Gamma$, and hence to any NEC group. However the second part, the use of character theory to count such homomorphisms, seems to be effective only for those NEC groups which contain no reflections. Several simple illustrative examples are considered, where $G$ is cyclic, dihedral, or of prime exponent. These methods are extensions of those used in [9], where $\Gamma$ is assumed to be a surface group, that is, it contains neither reflections nor elliptic elements (see also [10] for a general survey of applications of character theory to surfaces). Izquierdo has taken a complementary approach in [8], where reflections are allowed, $\Gamma$ has genus 0 , and $G$ is a dihedral group $D_{p}$ for some prime $p$; her methods are completely different, relying on the special structure of these dihedral groups.

One can interpret these enumerative results in terms of orbifold coverings (see [8] for a general account of this connection): if $\mathcal{O}$ is the orbifold $\mathscr{H} / \Gamma$ corresponding to $\Gamma$, where $\mathscr{H}$ is the hyperbolic plane, then $n_{\Gamma}(G)$ is the

Received August 19, 1996. 
number of equivalence classes of regular coverings of $\mathcal{O}$ with covering group $G$; the restriction that $\Gamma$ should have no reflections is equivalent to the condition that the underlying surface of $\mathcal{O}$ should be without boundary. By refining these techniques to count the normal surface subgroups of $\Gamma$ with quotient-group $G$, one can also count regular coverings of $\mathcal{O}$ by Klein surfaces, rather than by orbifolds. Similarly, a straightforward extension to non-normal subgroups of finite index allows one to count the non-regular finite coverings of $\mathcal{O}$ with a given permutation group as their monodromy group.

The author is grateful to the organisers and participants of the EU-funded Workshop on Computational Conformal Geometry, Helsinki, 1994, for valuable discussions which gave rise to this paper, and to the referee for some very helpful comments.

\section{Counting normal subgroups}

In [5], P. Hall developed a general technique for computing the number $n_{\Gamma}(G)$ of normal subgroups $N$ of a finitely generated group $\Gamma$ with a given finite quotient group $\Gamma / N \cong G$. These subgroups $N$ are the kernels of the epimorphisms $\theta: \Gamma \rightarrow G$; the set $\operatorname{Epi}(\Gamma, G)$ of such epimorphisms is finite (since there are only finitely many ways of mapping the generators of $\Gamma$ into $G)$, so $n_{\Gamma}(G)$ is finite. If $\theta_{1}, \theta_{2} \in \operatorname{Epi}(\Gamma, G)$, then $\operatorname{ker} \theta_{1}=\operatorname{ker} \theta_{2}$ if and only if $\theta_{2}=\theta_{1} \circ \alpha$ for some automorphism $\alpha$ of $G$, so $n_{\Gamma}(G)=|\operatorname{Epi}(\Gamma, G) / \operatorname{Aut} G|$, the number of orbits of Aut $G$ acting by composition on $\operatorname{Epi}(\Gamma, G)$. Since this action is fixed-point-free, every orbit has length $\mid$ Aut $G \mid$ and so

$$
n_{\Gamma}(G)=\frac{|\operatorname{Epi}(\Gamma, G)|}{|\operatorname{Aut} G|} .
$$

To count epimorphisms $\Gamma \rightarrow G$, one first counts the homomorphisms, and then eliminates those which map $\Gamma$ onto proper subgroups of $G$. One can invert the equation

$$
|\operatorname{Hom}(\Gamma, G)|=\sum_{K \leq G}|\operatorname{Epi}(\Gamma, K)|,
$$

to count epimorphisms in terms of homomorphisms, by introducing the Möbius function for $G$. This assigns an integer $\mu(K)$ to each subgroup $K$ of $G$ by the recursive formula

$$
\sum_{H \geq K} \mu(H)=\delta_{K, G}= \begin{cases}1 & \text { if } K=G, \\ 0 & \text { if } K<G .\end{cases}
$$


The equation

$$
|\operatorname{Epi}(\Gamma, G)|=\sum_{H \leq G} \mu(H)|\operatorname{Hom}(\Gamma, H)|
$$

is then easily deduced, and this immediately gives

$$
n_{\Gamma}(G)=\frac{1}{|\operatorname{Aut} G|} \sum_{H \leq G} \mu(H)|\operatorname{Hom}(\Gamma, H)| .
$$

For many groups $G$, it is a routine task to find $\mid$ Aut $G \mid$ and $\mu(H)$ for all $H \leq G$, so there remains the problem of computing $|\operatorname{Hom}(\Gamma, H)|$ for all $H \leq G$ (or at least, for all those $H \leq G$ with $\mu(H) \neq 0$ ). We shall use the character theory of finite groups to do this for various NEC groups $\Gamma$; for background information on NEC groups and Klein surfaces, see [1] or [14], for finite groups see [7], for character theory see [7] or [12], and for number theory see [6].

\section{Orientable NEC groups without reflections}

An NEC group $\Gamma$ is a discrete group of isometries of the hyperbolic plane $\mathscr{H}$, with compact quotient space $\mathscr{H} / \Gamma$. First let us take $\Gamma$ to be an orientable NEC group without reflections (that is, a co-compact Fuchsian group), so that $\Gamma$ has signature

$$
\left(g ;+;\left[m_{1}, \ldots, m_{r}\right] ;\{-\}\right)
$$

where $g, r, m_{1}, \ldots, m_{r}$ are integers with $g, r \geq 0$ and $m_{i}>1$ for all $i$. Here $g$ is the genus of $\mathscr{H} / \Gamma$, the symbol + denotes orientability, the integers $m_{i}$ are the periods of $\Gamma$, and $\{-\}$ denotes the absence of reflections. This means that $\Gamma$ has generators

$$
X_{i} \quad(i=1, \ldots, r), \quad A_{j}, B_{j} \quad(j=1, \ldots, g)
$$

and defining relations

$$
X_{i}^{m_{i}}=1 \quad(i=1, \ldots, r), \quad \prod_{i=1}^{r} X_{i} \cdot \prod_{j=1}^{g}\left[A_{j}, B_{j}\right]=1 .
$$

It follows that the number

$$
\sigma_{\Gamma}(H)=|\operatorname{Hom}(\Gamma, H)|
$$

of homomorphisms $\Gamma \rightarrow H$ is equal to the number of solutions $x_{i}, a_{j}, b_{j}$ in $H$ of the simultaneous equations 


$$
\begin{gathered}
x_{i}^{m_{i}}=1 \quad(i=1, \ldots, r), \\
\prod_{i=1}^{r} x_{i} \cdot \prod_{j=1}^{g}\left[a_{j}, b_{j}\right]=1 .
\end{gathered}
$$

This number can be computed by means of the following result, which is proved in [9]. (The case $r=0$, where $\Gamma$ is an orientable surface group, is due to Frobenius [3] for $g=1$, and to Mednykh [11] for $g>1$. See $§ 7.2$ of [13] for similar results.)

THEOREM 1. The number of solutions of equation $\left(2^{\prime \prime}\right)$ in a finite group $H$, where each $x_{i}$ lies in some union $L_{i}$ of conjugacy classes of $H$, is equal to

$$
|H|^{2 g-1} \sum_{\chi}\left\{\chi(1)^{2-2 g-r} \sum_{x_{1} \in L_{1}} \chi\left(x_{1}\right) \ldots \sum_{x_{r} \in L_{r}} \chi\left(x_{r}\right)\right\},
$$

where $\chi$ ranges over the irreducible complex characters of $H$.

Note that $\chi(1)$ is the degree of $\chi$, the dimension of the corresponding $\mathrm{CH}$ module. In order to deduce a formula for $\sigma_{\Gamma}(H)$, we will choose the sets $L_{i}$ to be the sets of the solutions $x_{i} \in H$ of equations $\left(2^{\prime}\right)$. First we need some notation. If $m$ is a positive integer, $H$ is a finite group and $\chi$ is a complex character of $H$, then let

$$
H[m]=\left\{h \in H \mid h^{m}=1\right\}
$$

(which is a union of conjugacy classes of $H$ ), and let

$$
\chi[m]=\sum_{h \in H[m]} \chi(h) .
$$

Then Theorem 1 immediately implies:

COROLlary 1. If $\Gamma$ has signature $(\mathrm{S}+)$ and $H$ is any finite group, then

$$
\sigma_{\Gamma}(H)=|H|^{2 g-1} \sum_{\chi} \chi(1)^{2-2 g-r} \chi\left[m_{1}\right] \cdots \chi\left[m_{r}\right],
$$

where $\chi$ ranges over the irreducible complex characters of $H$.

Given the character table of $H$, this result makes it straightforward to evaluate $\sigma_{\Gamma}(H)$. Character tables are available for many finite groups: see [2], for instance.

Before considering some examples, it is useful to introduce some notation: we let $l$ denote the least common multiple of the periods $m_{1}, \ldots, m_{r}$ of $\Gamma$, and for each integer $m$ we let $\nu_{m}$ be the number of $i$ such that $m$ divides $m_{i}$. 
EXAmples. (3.1) Let $H=C_{d}$, a cyclic group of order $d$. In this case, one can evaluate $\sigma_{\Gamma}(H)$ directly, without character theory, for instance by abelianising $\Gamma$ and using the structure theory of finitely-generated abelian groups. However, it is useful to apply Corollary 1 here as a simple illustration of the method.

There are $d$ irreducible characters $\chi$ of $H$, all of degree 1 ; these are the homomorphisms $H \rightarrow \mathrm{C}^{*}$, obtained by mapping a generator of $H$ to a $d$-th root of 1 in $\mathrm{C}$. If $m$ is any positive integer, then $H[m]$ is the unique subgroup of order $(m, d)$ in $H$, and so

$$
\chi[m]= \begin{cases}(m, d), & \text { if } H[m] \leq \operatorname{ker} \chi \\ 0, & \text { otherwise }\end{cases}
$$

It follows that a character $\chi$ makes a non-zero contribution to the formula for $\sigma_{\Gamma}(H)$ if and only if ker $\chi$ contains the subgroups $H\left[m_{i}\right]$ for all $i=1, \ldots, r$, or equivalently ker $\chi$ contains the subgroup $H[l]$ of order $(l, d)$ which they generate, where $l=\operatorname{lcm}\left(m_{1}, \ldots, m_{r}\right)$. There are $d /(l, d)$ such characters, each with $\chi\left[m_{i}\right]=\left(m_{i}, d\right)$, so

$$
\sigma_{\Gamma}\left(C_{d}\right)=\frac{d^{2 g}}{(l, d)} \cdot \prod_{i=1}^{r}\left(m_{i}, d\right) .
$$

For instance, if $d$ is a prime $p$ then

$$
\sigma_{\Gamma}\left(C_{p}\right)= \begin{cases}p^{2 g-1+\nu_{p}}, & \text { if } \nu_{p}>0, \\ p^{2 g}, & \text { if } \nu_{p}=0 .\end{cases}
$$

We can now compute $n_{\Gamma}(G)$ where $G=C_{n}$, a cyclic group of order $n$. First, we have $|\operatorname{Aut}(G)|=\phi(n)$, where $\phi$ is Euler's function on N. Now $G$ has a unique subgroup $H \cong C_{d}$ for each divisor $d$ of $n$, and has no other subgroups; for each such $H$ we have $\mu(H)=\mu(n / d)$, where $\mu$ on the right-hand side denotes the Möbius function on N. Equation (1) therefore gives

$$
\begin{aligned}
n_{\Gamma}\left(C_{n}\right) & =\frac{1}{\phi(n)} \sum_{d \mid n} \mu\left(\frac{n}{d}\right) \sigma_{\Gamma}\left(C_{d}\right) \\
& =\frac{1}{\phi(n)} \sum_{d \mid n}\left\{\mu\left(\frac{n}{d}\right) \frac{d^{2 g}}{(l, d)} \cdot \prod_{i=1}^{r}\left(m_{i}, d\right)\right\} .
\end{aligned}
$$


For example, if $n$ is a prime $p$ then we have

$$
n_{\Gamma}\left(C_{p}\right)= \begin{cases}\left(p^{2 g-1+\nu_{p}}-1\right) /(p-1) & \text { if } \nu_{p}>0 \\ \left(p^{2 g}-1\right) /(p-1) & \text { if } \nu_{p}=0\end{cases}
$$

(3.2) Let $H=D_{p}=\left\langle a, b \mid a^{p}=b^{2}=(a b)^{2}=1\right\rangle$, a dihedral group of order $2 p$, where $p$ is an odd prime. Apart from the identity element, this group has one conjugacy class consisting of the $p$ involutions $a^{i} b$, and $(p-1) / 2$ classes $\left\{a^{ \pm i}\right\}$ of elements of order $p$. In addition to the principal character $\chi_{1}$, the other irreducible characters $\chi$ of $H$ are the alternating character $\chi_{2}\left(a^{i} b^{j}\right)=(-1)^{j}$, together with $(p-1) / 2$ characters $\chi_{k}^{\prime}(k=1, \ldots,(p-1) / 2)$ given by $\chi_{k}^{\prime}\left(a^{i}\right)=\zeta^{i k}+\zeta^{-i k}$ and $\chi_{k}^{\prime}\left(a^{i} b\right)=0$, where $\zeta$ is a primitive $p$ th root of unity. It follows that

$$
\begin{aligned}
& \text { if } \quad(m, 2 p)=2 p \quad \text { then } \quad \chi[m]= \begin{cases}2 p & \text { if } \chi=\chi_{1}, \\
0 & \text { otherwise; }\end{cases} \\
& \text { if }(m, 2 p)=p \quad \text { then } \quad \chi[m]= \begin{cases}p & \text { if } \chi=\chi_{1} \text { or } \chi_{2}, \\
0 & \text { otherwise; }\end{cases} \\
& \text { if }(m, 2 p)=2 \quad \text { then } \quad \chi[m]= \begin{cases}p+1 \text { if } \chi=\chi_{1}, \\
1-p & \text { if } \chi=\chi_{2}, \\
2 & \text { otherwise; }\end{cases} \\
& \text { if }(m, 2 p)=1 \quad \text { then } \quad \chi[m]= \begin{cases}1 & \text { if } \chi=\chi_{1} \text { or } \chi_{2}, \\
2 & \text { otherwise. }\end{cases}
\end{aligned}
$$

If $n_{h}$ denotes the number of $i$ such that $\left(m_{i}, 2 p\right)=h$ then Corollary 1 gives

$$
\begin{aligned}
\sigma_{\Gamma}\left(D_{p}\right)= & (2 p)^{2 g-1}\left\{(2 p)^{n_{2 p}} \cdot p^{n_{p}} \cdot(p+1)^{n_{2}}+0^{n_{2 p}} \cdot p^{n_{p}} \cdot(1-p)^{n_{2}}\right. \\
& \left.\quad+\frac{p-1}{2} \cdot 2^{2-2 g-r} \cdot 0^{n_{2 p}} \cdot 0^{n_{p}} \cdot 2^{n_{2}+n_{1}}\right\} \\
= & (2 p)^{2 g-1}\left\{2^{\nu_{2 p}} \cdot p^{\nu_{p}} \cdot(p+1)^{n_{2}}+0^{n_{2 p}} \cdot p^{n_{p}} \cdot(1-p)^{n_{2}}+\frac{p-1}{2} \cdot 0^{\nu_{p}} \cdot 2^{2-2 g-\nu_{p}}\right\},
\end{aligned}
$$

where we interpret $0^{0}$ as meaning 1 .

If we take $G=D_{p}$ then the subgroups $H \leq G$ are $H=D_{p}$, a unique subgroup $H=\langle a\rangle$ isomorphic to $C_{p}, p$ subgroups $\left\langle a^{i} b\right\rangle \cong C_{2}$, and the trivial subgroup $C_{1}$. The values of $\mu(H)$ for these subgroups are $1,-1,-1$ and $p$ respectively, and $\left|\operatorname{Aut}\left(D_{p}\right)\right|=p(p-1)$, so equation (1) gives

$$
n_{\Gamma}\left(D_{p}\right)=\frac{1}{p(p-1)}\left\{\sigma_{\Gamma}\left(D_{2 p}\right)-\sigma_{\Gamma}\left(C_{p}\right)-p \sigma_{\Gamma}\left(C_{2}\right)+p \sigma_{\Gamma}\left(C_{1}\right)\right\} \text {. }
$$


We have just computed $\sigma_{\Gamma}(H)$ for $H=D_{p}$, and Example 3.1 deals with the remaining subgroups $H$, so one can substitute these values and obtain $n_{\Gamma}\left(D_{p}\right)$. As in most cases where $|G|$ is divisible by more than one prime, the resulting general formula is rather unwieldy, so we will omit it and instead give a simple example. Suppose that $\Gamma$ has signature $(S+)$ with $m_{1}=\cdots=m_{r}=p$ and $r \geq 1$; then we find that $\sigma_{\Gamma}(H)=2^{2 g} p^{2 g-1+r}, p^{2 g-1+r}, 2^{2 g}$ and 1 for $H=D_{p}, C_{p}, C_{2}$ and 1 respectively, so

$$
n_{\Gamma}\left(D_{p}\right)=\frac{\left(2^{2 g}-1\right)\left(p^{2 g-2+r}-1\right)}{p-1} .
$$

(This formula is also valid in the case $r=0$, as shown in [9].)

(3.3) If $H$ has prime exponent $p$ then $H[m]=H$ or 1 as $p$ does or does not divide $m$. In the first case the orthogonality relations for the characters of $H$ give $\chi[m]=|H|$ or 0 as $\chi=\chi_{1}$ (the principal character) or $\chi \neq \chi_{1}$, and in the second case each character satisfies $\chi[m]=\chi(1)$, the degree of $\chi$. It follows that if $\nu_{p}>0$ (so that $p \mid m_{i}$ for some $i$ ) then only $\chi_{1}$ contributes to $\sigma_{\Gamma}(H)$, and we have

$$
\sigma_{\Gamma}(H)=|H|^{2 g-1+\nu_{p}} ;
$$

if $\nu_{p}=0$, on the other hand, then $\chi\left[m_{i}\right]=\chi(1)$ for all $\chi$ and all $i$, so that

$$
\sigma_{\Gamma}(H)=|H|^{2 g-1} \sum_{\chi} \chi(1)^{2-2 g} .
$$

(In particular, if $H$ is an elementary abelian $p$-group $C_{p} \times \cdots \times C_{p}$, so that there are $|H|$ characters $\chi$ of degree 1 , then

$$
\sigma_{\Gamma}(H)= \begin{cases}|H|^{2 g-1+\nu_{p}}, & \text { if } \nu_{p}>0, \\ |H|^{2 g}, & \text { if } \nu_{p}=0 ;\end{cases}
$$

this generalises the result in Example 3.1 for $H=C_{p}$.)

If $G$ has exponent $p$ then so does every non-trivial subgroup $H \leq G$, and one can apply these formulæ, together with the values of $|\operatorname{Aut}(G)|$ and $\mu(H)$, to determine $n_{\Gamma}(G)$. For example, let $G$ be the unique non-abelian group of order $p^{3}$ and exponent $p$ (where $p>2$ ). Apart from $G$ itself, the subgroups $H \leq G$ with $\mu(H) \neq 0$ are the $p+1$ maximal subgroups, all isomorphic to $C_{p} \times C_{p}$ and satisfying $\mu(H)=-1$, together with their intersection (the Frattini subgroup $\Phi(G) \cong C_{p}$, also equal to the centre $Z(G)$ ), for which $\mu(H)=p$. It follows from the Burnside Basis Theorem ([7], III.3.15) that a pair of elements generate $G$ if and only if their images generate the Frattini 
factor group $G / \Phi(G) \cong C_{p} \times C_{p}$; any two such pairs are equivalent under a unique automorphism of $G$, so by counting such pairs we see that $\operatorname{Aut}(G)$ has order $\left(p^{3}-p\right)\left(p^{3}-p^{2}\right)=p^{3}\left(p^{2}-1\right)(p-1)$. Thus equation (1) becomes

$$
n_{\Gamma}(G)=\frac{1}{p^{3}\left(p^{2}-1\right)(p-1)}\left\{\sigma_{\Gamma}(G)-(p+1) \sigma_{\Gamma}\left(C_{p} \times C_{p}\right)+p \sigma_{\Gamma}\left(C_{p}\right)\right\} .
$$

Since $G$ has $p^{2}$ irreducible characters of degree 1 , and $p-1$ of degree $p$, Corollary 1 gives

$$
\sigma_{\Gamma}(G)= \begin{cases}p^{3\left(2 g-1+\nu_{p}\right)}, & \text { if } \nu_{p}>0 \\ p^{3(2 g-1)}\left(p^{2}+(p-1) p^{2-2 g}\right), & \text { if } \nu_{p}=0 .\end{cases}
$$

We have already evaluated $\sigma_{\Gamma}(H)$ for the elementary abelian groups $H=C_{p} \times C_{p}$ and $C_{p}$, so writing $2 g-1+\nu_{p}=h$ we obtain

$$
n_{\Gamma}(G)= \begin{cases}p^{h-2}\left(p^{h}-1\right)\left(p^{h-1}-1\right) /\left(p^{2}-1\right)(p-1), & \text { if } \nu_{p}>0, \\ p^{h-1}\left(p^{h+1}-1\right)\left(p^{h-1}-1\right) /\left(p^{2}-1\right)(p-1), & \text { if } \nu_{p}=0 .\end{cases}
$$

\section{Non-orientable NEC groups without reflections}

Now let $\Gamma$ be an non-orientable NEC group without reflections, so that $\Gamma$ has signature

$$
\left(g ;-;\left[m_{1}, \ldots, m_{r}\right] ;\{-\}\right)
$$

where $g, r, m_{1}, \ldots, m_{r}$ are integers with $g \geq 1, r \geq 0$ and $m_{i}>1$ for all $i$. Thus $\Gamma$ has generators

$$
X_{i} \quad(i=1, \ldots, r), \quad A_{j} \quad(j=1, \ldots, g)
$$

and defining relations

$$
X_{i}^{m_{i}}=1 \quad(i=1, \ldots, r), \quad \prod_{i=1}^{r} X_{i} \cdot \prod_{j=1}^{g} A_{j}^{2}=1 .
$$

In this case, the number

$$
\sigma_{\Gamma}(H)=|\operatorname{Hom}(\Gamma, H)|
$$

of homomorphisms $\Gamma \rightarrow H$ is equal to the number of solutions $x_{i}, a_{j}$ in $H$ of the simultaneous equations

$$
x_{i}^{m_{i}}=1 \quad(i=1, \ldots, r),
$$




$$
\prod_{i=1}^{r} x_{i} \cdot \prod_{j=1}^{g} a_{j}^{2}=1
$$

The formula for $\sigma_{\Gamma}(H)$ is similar to that in the orientable case, but it contains one extra ingredient. Let $\chi$ be an irreducible character of $H$, afforded by a representation $\rho$. The Frobenius-Schur indicator of $\chi$ (or of $\rho$ ) is defined to be

$$
c_{\chi}=\frac{1}{|H|} \sum_{h \in H} \chi\left(h^{2}\right) \text {. }
$$

We have

$$
c_{\chi}= \begin{cases}1, & \text { if } \rho \text { is real, } \\ -1, & \text { if } \chi \text { is real but } \rho \text { is not real, } \\ 0, & \text { if } \chi \text { is not real. }\end{cases}
$$

The following result is proved in [9]; the case $r=0$ is due to Frobenius and Schur [4].

THEOREM 2. The number of solutions of equation $\left(3^{\prime \prime}\right)$ in a finite group $H$, where each $x_{i}$ lies in some union $L_{i}$ of conjugacy classes of $H$, is equal to

$$
|H|^{g-1} \sum_{\chi}\left\{c_{\chi}^{g} \chi(1)^{2-g-r} \sum_{x_{1} \in L_{1}} \chi\left(x_{1}\right) \ldots \sum_{x_{r} \in L_{r}} \chi\left(x_{r}\right)\right\}
$$

where $\chi$ ranges over the irreducible complex characters of $H$.

COROLlary 2. If $\Gamma$ has signature $(\mathrm{S}-)$ and $H$ is any finite group, then

$$
\sigma_{\Gamma}(H)=|H|^{g-1} \sum_{\chi} c_{\chi}^{g} \chi(1)^{2-g-r} \chi\left[m_{1}\right] \cdots \chi\left[m_{r}\right],
$$

where $\chi$ ranges over the irreducible complex characters of $H$.

EXAmPles. (4.1) Let $H=C_{d}$, a cyclic group of order $d$. By our earlier description of the characters $\chi$ of $H$, we see that $c_{\chi}=0$ unless either $\chi=\chi_{1}$, the principal character given by $\chi(h)=1$ for all $h$, or else $d$ is even and $\chi=\chi_{2}$, the alternating character which maps a generator of $H$ to -1 . Both of these characters have $c_{\chi}=1$, so a similar argument to that in the orientable case gives

$$
\sigma_{\Gamma}\left(C_{d}\right)=\delta d^{g-1} \cdot \prod_{i=1}^{g}\left(m_{i}, d\right)
$$

where 


$$
\delta= \begin{cases}1, & \text { if } d /(l, d) \text { is odd, } \\ 2, & \text { if } d /(l, d) \text { is even, }\end{cases}
$$

and $l$ is the least common multiple of the periods $m_{i}$. Thus if $(m)_{2}$ denotes the highest power of 2 dividing an integer $m$, then

$$
\delta= \begin{cases}1, & \text { if }\left(m_{i}\right)_{2} \geq(d)_{2} \text { for some } i \\ 2, & \text { if }\left(m_{i}\right)_{2}<(d)_{2} \text { for all } i\end{cases}
$$

For instance, if we take $d$ to be an odd prime $p$ we obtain

$$
\sigma_{\Gamma}\left(C_{p}\right)=p^{g-1+\nu_{p}}
$$

whereas by taking $d=2$ we have

$$
\sigma_{\Gamma}\left(C_{2}\right)= \begin{cases}2^{g-1+\nu_{2}}, & \text { if } \nu_{2}>0 \\ 2^{g}, & \text { if } \nu_{2}=0\end{cases}
$$

(4.2) The calculation of $\sigma_{\Gamma}\left(D_{p}\right)$ is similar to that in the orientable case (Example 3.2): the values of $\chi[m]$ are unchanged, and since every irreducible representation of a dihedral group is real, we have $c_{\chi}=1$ for all $\chi$. From this, one can deduce a formula for $n_{\Gamma}\left(D_{p}\right)$ : equation (1) is the same as before, and Example 4.1 gives $\sigma_{\Gamma}(H)$ for the proper subgroups $H$ of $D_{p}$. For a simple example, suppose that $m_{1}=\cdots=m_{r}=p$ and $r \geq 1$. Then $\sigma_{\Gamma}(H)=2^{g} p^{g-1-r}, p^{g-1-r}, 2^{g}$ and 1 for $H=D_{p}, C_{p}, C_{2}$ and $C_{1}$ respectively, so

$$
n_{\Gamma}\left(D_{p}\right)=\frac{\left(2^{g}-1\right)\left(p^{g-2-r}-1\right)}{p-1} .
$$

(This formula is the same as that obtained in the orientable case, except that there each exponent $g$ is replaced with $2 g$.)

(4.3) Let $H$ have prime exponent $p$. We can use the values of $\chi[m]$ which we determined in Example 3.3. If $\nu_{p}>0$ then since $c_{\chi}=1$ for the principal character $\chi=\chi_{1}$, we have

$$
\sigma_{\Gamma}(H)=|H|^{g-1+\nu_{p}}
$$

if $\nu_{p}=0$, however, then

$$
\sigma_{\Gamma}(H)=|H|^{g-1} \sum_{\chi} c_{\chi}^{g} \chi(1)^{2-g} .
$$

In this latter case, if $p$ is odd then $c_{\chi}=0$ for all $\chi \neq \chi_{1}$, so that 


$$
\sigma_{\Gamma}(H)=|H|^{g-1}=|H|^{g-1+\nu_{p}},
$$

as before, whereas if $p=2$ then $c_{\chi}=1$ and $\chi(1)=1$ for every $\chi$ (since $H$ is abelian), so that

$$
\sigma_{\Gamma}(H)=|H|^{g} .
$$

As in the orientable case, these results extend those in Example 4.1 for $H=C_{p}$. One can now compute $n_{\Gamma}(G)$ when $G$ has exponent $p$. For instance, if $G$ is the non-abelian group of order $p^{3}$ and exponent $p$ (where $p>2$ ), then as before we have

$$
n_{\Gamma}(G)=\frac{1}{p^{3}\left(p^{2}-1\right)(p-1)}\left\{\sigma_{\Gamma}(G)-(p+1) \sigma_{\Gamma}\left(C_{p} \times C_{p}\right)+p \sigma_{\Gamma}\left(C_{p}\right)\right\} .
$$

Since $p$ is odd, the above results give $\sigma_{\Gamma}(H)=|H|^{h}$ for each subgroup $H \leq G$ on the right-hand side, where $h=g-1+\nu_{p}$, so a little algebra yields

$$
n_{\Gamma}(G)=\frac{p^{h-2}\left(p^{h}-1\right)\left(p^{h-1}-1\right)}{\left(p^{2}-1\right)(p-1)} .
$$

\section{Normal surface subgroups}

Since $\Gamma$ contains no reflections, its torsion elements are the conjugates of the powers of the elliptic generators $X_{i}$. Thus a normal subgroup $N$ of $\Gamma$ is torsion-free if and only if it contains no non-trivial powers of any $X_{i}$, that is, each $X_{i}$ is mapped to an element $x_{i}$ of order exactly $m_{i}$ in the quotient-group $G=\Gamma / N$. This is equivalent to $N$ being a surface group (orientable or nonorientable), or equivalently the orbifold $\mathscr{H} / N$ being a surface (without conepoints). In fact, in this situation $\mathscr{H} / N$ is a Klein surface without boundary, its dianalytic structure being induced by projection from $\mathscr{H}$; conversely, every compact Klein surface without boundary, other than the sphere, projective plane, torus and Klein bottle, arises in this way (these exceptional surfaces have spherical or euclidean uniformisations). One can apply Hall's theory as before to show that the number $n_{\Gamma}^{s}(G)$ of normal surface subgroups $N$ in $\Gamma$, with $\Gamma / N \cong G$, is given by

$$
n_{\Gamma}^{s}(G)=\frac{1}{|\operatorname{Aut} G|} \sum_{H \leq G} \mu(H) \sigma_{\Gamma}^{s}(H),
$$

where $\sigma_{\Gamma}^{s}(H)$ denotes the number of surface-kernel homomorphisms $\theta: \Gamma \rightarrow H$; these are the homomorphisms with torsion-free kernel, that is, such that $x_{i}=X_{i} \theta$ has order $m_{i}$ for each $i=1, \ldots, r$. 
For each finite group $H$ and each integer $m \geq 1$, let $H\langle m\rangle$ denote the set of elements of order $m$ in $H$, and for each character $\chi$ of $H$ let

$$
\chi\langle m\rangle=\sum_{h \in H\langle m\rangle} \chi(h) .
$$

Theorems 1 and 2 immediately imply the two parts of the following result.

COROllary 3. Let $H$ be any finite group. If $\Gamma$ has signature $(\mathrm{S}+)$ then

$$
\sigma_{\Gamma}^{s}(H)=|H|^{2 g-1} \sum_{\chi} \chi(1)^{2-2 g-r} \chi\left\langle m_{1}\right\rangle \cdots \chi\left\langle m_{r}\right\rangle,
$$

and if $\Gamma$ has signature $(\mathrm{S}-)$ then

$$
\sigma_{\Gamma}^{s}(H)=|H|^{g-1} \sum_{\chi} c_{\chi}^{g} \chi(1)^{2-g-r} \chi\left\langle m_{1}\right\rangle \cdots \chi\left\langle m_{r}\right\rangle
$$

where in each case, $\chi$ ranges over the irreducible complex characters of $H$.

ExAmples. (5.1) Let us calculate $n_{\Gamma}^{s}\left(C_{n}\right)$. All subgroups $H$ of $C_{n}$ are cyclic, so first let $H=C_{d}$, let $h$ be any generator of $H$, and let $\zeta$ be a primitive $d$-th root of 1 in $\mathrm{C}$. Then the irreducible characters $\chi$ of $H$ are the homomorphisms $\lambda_{j}: H \rightarrow S^{1} \subset \mathrm{C}$, determined by mapping $h$ to $\zeta^{j}(j=1, \ldots, d)$; the kernel of $\chi=\lambda_{j}$ has order $k=(j, d)$, and the image has order $d / k$. If $m$ does not divide $d$ then $\chi\langle m\rangle=0$, so assume that $m \mid d$. Then $H\langle m\rangle$ consists of the elements of $H$ which are generators of its unique subgroup $C_{m}$. Now $C_{m} \cap \operatorname{ker} \chi=C_{(m, k)}$, so $\chi$ maps $C_{m}$ onto the group of $m /(m, k)$-th roots of unity, sending generators to primitive roots; each primitive root is the image of $\phi(m) / \phi(m /(m, k))$ elements of $H\langle m\rangle$, and since the sum of the primitive $n$-th roots of 1 in $\mathrm{C}$ is $\mu(n)$ for all $n$, we find that

$$
\chi\langle m\rangle=\frac{\phi(m) \mu(m /(m, k))}{\phi(m /(m, k))},
$$

which we will abbreviate to

$$
\phi(m)\left(\frac{\mu}{\phi}\right)\left(\frac{m}{(m, k)}\right) .
$$

(This is the Ramanujan sum $c_{m}(j)$, the sum of the $j$-th powers of the primitive $m$-th roots of 1 , given by

$$
c_{m}(j)=\phi(m)\left(\frac{\mu}{\phi}\right)\left(\frac{m}{(m, j)}\right)
$$


where $(m, k)=(m,(j, d))=(m, j, d)=(m, j)$ since $m \mid d$; see [6, $\S \S 5.6,16.6]$ for details.)

Now let $\Gamma$ have signature $(\mathrm{S}+)$. If $\sigma_{\Gamma}^{s}\left(C_{d}\right)>0$ then each period $m_{i}$ must divide $d$, and hence their least common multiple $l$ must also divide $d$. If we assume that $l$ divides $d$, then the above argument gives

$$
\begin{aligned}
\sigma_{\Gamma}^{s}\left(C_{d}\right) & =d^{2 g-1} \sum_{\chi}\left\{\prod_{i=1}^{r} \phi\left(m_{i}\right)\left(\frac{\mu}{\phi}\right)\left(\frac{m_{i}}{\left(m_{i}, k\right)}\right)\right\} \\
& =d^{2 g-1} \prod_{i=1}^{r} \phi\left(m_{i}\right) \cdot \sum_{\chi}\left\{\prod_{i=1}^{r}\left(\frac{\mu}{\phi}\right)\left(\frac{m_{i}}{\left(m_{i}, k\right)}\right)\right\} \\
& =d^{2 g-1} \prod_{i=1}^{r} \phi\left(m_{i}\right) \cdot \sum_{k \mid d}\left\{\phi\left(\frac{d}{k}\right) \prod_{i=1}^{r}\left(\frac{\mu}{\phi}\right)\left(\frac{m_{i}}{\left(m_{i}, k\right)}\right)\right\},
\end{aligned}
$$

where we have used the fact that there are $\phi(d / k)$ irreducible characters $\chi$ with $|\operatorname{ker} \chi|=k$ for each $k$ dividing $d$.

If we take $G=C_{n}$ then all its subgroups $H$ are cyclic groups $C_{d}$; we can therefore substitute the above formula for $\sigma_{\Gamma}^{s}(H)$ in equation (4), giving

$$
\begin{aligned}
n_{\Gamma}^{s}\left(C_{n}\right) & =\frac{1}{\phi(n)} \sum_{d \mid n} \mu\left(\frac{n}{d}\right) \sigma_{\Gamma}^{s}\left(C_{d}\right) \\
& =\frac{1}{\phi(n)} \sum_{l|d| n}\left\{\mu\left(\frac{n}{d}\right) d^{2 g-1} \prod_{i=1}^{r} \phi\left(m_{i}\right) . \sum_{k \mid d}\left\{\phi\left(\frac{d}{k}\right) \prod_{i=1}^{r}\left(\frac{\mu}{\phi}\right)\left(\frac{m_{i}}{\left(m_{i}, k\right)}\right)\right\}\right\} \\
& =\frac{1}{\phi(n)} \prod_{i=1}^{r} \phi\left(m_{i}\right) \sum_{l|d| n}\left\{\mu\left(\frac{n}{d}\right) d^{2 g-1} \sum_{k \mid d}\left\{\phi\left(\frac{d}{k}\right) \prod_{i=1}^{r}\left(\frac{\mu}{\phi}\right)\left(\frac{m_{i}}{\left(m_{i}, k\right)}\right)\right\}\right\} .
\end{aligned}
$$

For example, let $n$ be a prime $p$. If $r \geq 1$ then the main sum is empty (and so $n_{\Gamma}^{s}\left(C_{p}\right)=0$ ) unless $l=p$, that is, $m_{i}=p$ for each $i$. In this case, the only possible value for $d$ in the summation is $d=p$, so $k=1$ or $p$, and we find that

$$
n_{\Gamma}^{s}\left(C_{p}\right)=p^{2 g-1}\left((p-1)^{r-1}+(-1)^{r}\right) .
$$

If $r=0$, on the other hand, then $l=1$, so $d=1$ or $p$, and we get

$$
n_{\Gamma}^{s}\left(C_{p}\right)=\frac{p^{2 g}-1}{p-1} .
$$

(This agrees with our earlier value for $n_{\Gamma}\left(C_{p}\right)$, since $\Gamma$ is torsion-free when $r=0$.) 
The calculation of $\sigma_{\Gamma}^{s}\left(C_{d}\right)$ is a little simpler in the non-orientable case, since the only irreducible characters $\chi$ of $C_{d}$ with $c_{\chi} \neq 0$ are the principal character $\chi_{1}$ and the alternating character $\chi_{2}$ (when $d$ is even); these two characters, which send a generator of $C_{d}$ to \pm 1 respectively, satisfy $c_{\chi}=1$. As before, we have $\chi\langle m\rangle=0$ unless $m$ divides $d$, in which case $\chi_{1}\langle m\rangle=\phi(m)$ and (if $d$ is even) $\chi_{2}\langle m\rangle=(-1)^{d / m} \phi(m)$. It follows that if $\omega_{d}$ denotes the number of $i$ such that $d / m_{i}$ is odd, then

$$
\sigma_{\Gamma}^{s}\left(C_{d}\right)= \begin{cases}d^{g-1} \phi\left(m_{1}\right) \ldots \phi\left(m_{r}\right), & \text { if } l \mid d \text { and } d \text { is odd } \\ 2 d^{g-1} \phi\left(m_{1}\right) \ldots \phi\left(m_{r}\right), & \text { if } l \mid d, d \text { is even and } \omega_{d} \text { is even } \\ 0, & \text { otherwise }\end{cases}
$$

so equation (4) gives

$$
\begin{aligned}
n_{\Gamma}^{s}\left(C_{n}\right) & =\frac{1}{\phi(n)}\left\{\sum_{\substack{l|d| n \\
d \text { odd }}} \mu\left(\frac{n}{d}\right) d^{g-1} \prod_{i=1}^{r} \phi\left(m_{i}\right)+\sum_{\substack{l|d| n \\
d, \omega_{d} \text { even }}} 2 \mu\left(\frac{n}{d}\right) d^{g-1} \prod_{i=1}^{r} \phi\left(m_{i}\right)\right\} \\
& =\frac{1}{\phi(n)} \prod_{i=1}^{r} \phi\left(m_{i}\right)\left\{\sum_{\substack{l|d| n \\
d \text { odd }}} \mu\left(\frac{n}{d}\right) d^{g-1}+\sum_{\substack{l|d| n \\
d, \omega_{d} \text { even }}} 2 \mu\left(\frac{n}{d}\right) d^{g-1}\right\} .
\end{aligned}
$$

For example, let $n$ be an odd prime $p$. If $r \geq 1$ then $n_{\Gamma}^{s}\left(C_{p}\right)=0$ unless $l=p$, that is, $m_{i}=p$ for all $i$, in which case $n_{\Gamma}^{s}\left(C_{p}\right)=(p-1)^{r-1} p^{g-1}$; if $r=0$ (so that $l=1$ ) then $n_{\Gamma}^{s}\left(C_{p}\right)=\left(p^{g-1}-1\right) /(p-1)$. Similarly, if $n=p=2$ and $r \geq 1$ we get $n_{\Gamma}^{s}\left(C_{2}\right)=2^{g}$ or 0 as $r$ is even or odd, while for $r=0$ we get $n_{\Gamma}^{s}\left(C_{2}\right)=2^{g}-1$. As in the orientable case, one can confirm these results by considering epimorphisms from the finite abelian group $\Gamma / \Gamma^{\prime} \Gamma^{p}$ onto $C_{p}$.

(5.2) Let $G=D_{p}$, where $p$ is an odd prime, and suppose that $\Gamma$ has periods $m_{1}=\cdots=m_{r}=p$ with $r \geq 1$. A surface-kernel homomorphism $\Gamma \rightarrow D_{p}$ must have image $H \leq D_{p}$ containing elements $x_{i}$ of order $p$, so $H=C_{p}$ or $D_{p}$ and hence

$$
n_{\Gamma}^{s}\left(D_{p}\right)=\frac{1}{p(p-1)}\left(\sigma_{\Gamma}^{s}\left(D_{p}\right)-\sigma_{\Gamma}^{s}\left(C_{p}\right)\right) .
$$

If $\Gamma$ is orientable, then Example 5.1 shows that

$$
\sigma_{\Gamma}^{s}\left(C_{p}\right)=p^{2 g-1}(p-1)\left((p-1)^{r-1}+(-1)^{r}\right) .
$$

The two 1-dimensional characters $\chi$ of $D_{p}$ satisfy $\chi\langle p\rangle=p-1$, while the $(p-1) / 2$ remaining characters (all 2-dimensional) satisfy $\chi\langle p\rangle=-2$, so Corollary 3 gives 
$\sigma_{\Gamma}^{s}\left(D_{p}\right)=(2 p)^{2 g-1} \sum_{\chi} \chi(1)^{2-2 g-r} \chi\langle p\rangle^{r}=p^{2 g-1}(p-1)\left(2^{2 g}(p-1)^{r-1}+(-1)^{r}\right)$,

from which we obtain

$$
n_{\Gamma}^{s}\left(D_{p}\right)=p^{2 g-2}(p-1)^{r-1}\left(2^{2 g}-1\right) .
$$

Similarly, in the non-orientable case we find that $\sigma_{\Gamma}^{s}\left(C_{p}\right)=(p-1)^{r} p^{g-1}$ and $\sigma_{\Gamma}^{s}\left(D_{p}\right)=2 p^{g-1}(p-1)\left(2^{g-1}(p-1)^{r-1}+(-1)^{r}\right)$, so

$$
n_{\Gamma}^{s}\left(D_{p}\right)=p^{g-2}\left(\left(2^{g}-1\right)(p-1)^{r-1}+2(-1)^{r}\right) .
$$

\section{Non-normal subgroups and monodromy groups}

It is possible to extend the method of this paper so that it applies to non-normal subgroups $M$ of finite index in an NEC group $\Gamma$, with $\Gamma$ inducing a given permutation group on their cosets. This is because the core of $M$ (the intersection of its conjugates in $\Gamma$ ) is a normal subgroup $N$ of finite index in $\Gamma$, and $\Gamma / N$ is isomorphic to the finite transitive permutation group $G$ induced by $\Gamma$ acting on the cosets of $M$. The details are as follows.

Let $G$ be a finite group with a faithful, transitive permutation representation on a set $\Omega$. Our aim is to determine the number $n_{\Gamma}(G, \Omega)$ of subgroups $M$ of $\Gamma$ on whose cosets $\Gamma$ induces a permutation group $(\Gamma / N, \Gamma / M)$ isomorphic to $(G, \Omega)$. (An isomorphism between permutation groups $(G, \Omega$ ) and $\left(G^{\prime}, \Omega^{\prime}\right)$ consists of an isomorphism $G \rightarrow G^{\prime}, g \mapsto g^{\prime}$ and a bijection $\Omega \rightarrow \Omega^{\prime}, \omega \mapsto \omega^{\prime}$ such that $(\omega g)^{\prime}=\omega^{\prime} g^{\prime}$ for all $\omega \in \Omega$ and $g \in G$.) This extends our earlier enumeration of normal subgroups, corresponding to the regular representation of $G$.

Firstly, the techniques of $\$ \S 2-4$ are used to find the number $n_{\Gamma}(G)$ of normal subgroups $N$ of $\Gamma$ with quotient-group $\Gamma / N$ isomorphic to the abstract group $G$. For each such core $N$, the subgroups $M \geq N$ on whose cosets $\Gamma$ induces a permutation group isomorphic to $(G, \Omega)$ are in one-to-one correspondence with the point-stabilisers $G_{\omega}=\{g \in G \mid \omega g=\omega\}(\omega \in \Omega)$ in such representations of $G$. Now these stabilisers form an orbit under $A=$ Aut $G$, so the number of them is equal to the index $\left|A: N_{A}\left(G_{\omega}\right)\right|$ in $A$ of their normalisers $N_{A}\left(G_{\omega}\right)=\left\{\alpha \in A \mid\left(G_{\omega}\right) \alpha=G_{\omega}\right\}$, and hence

$$
n_{\Gamma}(G, \Omega)=\left|A: N_{A}\left(G_{\omega}\right)\right| . n_{\Gamma}(G) .
$$

The permutation group $(G, \Omega)$ is isomorphic to the monodromy group of the orbifold covering $\mathscr{H} / M \rightarrow \mathcal{O}=\mathscr{H} / \Gamma$, that is, the group of permutations of the sheets induced by lifting closed paths in $\mathcal{O}$. It follows therefore that 
this method enumerates the coverings of $\mathcal{O}$ with a given permutation group as their monodromy group.

To determine how many of these coverings are by Klein surfaces, one restricts this enumeration to surface subgroups $M$, as in $§ 5$. A subgroup $M$ is a surface group if and only if it contains no elliptic elements, that is, each of the generators $x_{i}(i=1, \ldots, r)$ of $G$ acts on $\Omega$ as a semi-regular permutation of order $m_{i}$, consisting entirely of cycles of length $m_{i}$. One can therefore find the number $n_{\Gamma}^{s}(G, \Omega)$ of surface subgroups $M$, on whose cosets $\Gamma$ induces $(G, \Omega)$, by taking each $L_{i}$ in Theorem 1 or 2 to be the set of elements of order $m_{i}$ in $G$ which act semi-regularly on $\Omega$.

EXAmple (6.1). Let $G=D_{p}$ acting naturally on the set $\Omega$ of vertices of a regular $p$-gon, where $p$ is an odd prime. A point-stabiliser $G_{\omega}\left(\cong C_{2}\right)$ lies in an orbit of length $p$ under $A=$ Aut $D_{p}$, so equation (5) gives $n_{\Gamma}\left(D_{p}, \Omega\right)=p n_{\Gamma}\left(D_{p}\right)$; the calculation of $n_{\Gamma}\left(D_{p}\right)$ was discussed in Examples 3.2 and 4.2 in the orientable and non-orientable cases. To determine $n_{\Gamma}^{s}\left(D_{p}, \Omega\right)$ one needs to know the non-identity elements of $D_{p}$ which are semiregular on $\Omega$; these are the rotations $a, a^{2}, \ldots, a^{p-1}$, which coincide with the elements of order $p$, so if some $m_{i} \neq p$ then $n_{\Gamma}^{s}\left(D_{p}, \Omega\right)=0$. Let us therefore assume that $m_{i}=p$ for $i=1, \ldots, r$. We may also assume that $r \geq 1$, for otherwise $n_{\Gamma}^{s}\left(D_{p}, \Omega\right)=n_{\Gamma}\left(D_{p}, \Omega\right)$ and there is no problem. In the orientable case, Example 5.2 shows that there are $n_{\Gamma}^{s}\left(D_{p}\right)=p^{2 g-2}(p-1)^{r-1}\left(2^{2 g}-1\right)$ possible kernels $N$; each is the core of $p$ surface subgroups $M$, so

$$
n_{\Gamma}^{s}\left(D_{p}, \Omega\right)=p^{2 g-1}(p-1)^{r-1}\left(2^{2 g}-1\right) .
$$

Similarly, in the non-orientable case there are

$$
n_{\Gamma}^{s}\left(D_{p}\right)=p^{g-2}\left(\left(2^{g}-1\right)(p-1)^{r-1}+2(-1)^{r}\right)
$$

kernels $N$ and hence there are

$$
n_{\Gamma}^{s}\left(D_{p}, \Omega\right)=p^{g-1}\left(\left(2^{g}-1\right)(p-1)^{r-1}+2(-1)^{r}\right)
$$

surface subgroups $M$. In either case, each conjugacy class of these subgroups $M$ consists of all those with a given core $N$, so the number of conjugacy classes, and hence the number of equivalence classes of surface coverings of (O) with monodromy group $\left(D_{p}, \Omega\right)$, is given by the above formulæ for $n_{\Gamma}^{s}\left(D_{p}\right)$. 


\section{REFERENCES}

1 E. Bujalance, J. J. Etayo, J. M. Gamboa and G. Gromadzki, Automorphism Groups of Compact Bordered Klein Surfaces, Lecture Notes in Math. 1439, 1990.

2 J. H. Conway, R. T. Curtis, S. P. Norton, R. A. Parker and R. A. Wilson, ATLAS of Finite Groups, Clarendon Press, Oxford, 1985.

3 G. Frobenius, Über Gruppencharaktere, Sitzber. Königlich Preuss. Akad. Wiss. Berlin (1896), 985-1021.

4 G. Frobenius and I. Schur, Über die reellen Darstellungen der endlichen Gruppen, Sitzber. Königlich Preuss. Akad. Wiss. Berlin (1906), 186-208.

5 P. Hall, The Eulerian functions of a group, Quarterly J. Math. Oxford 7 (1936), 134-151.

6 G. H. Hardy and E. M. Wright, Introduction to the Theory of Numbers (5th ed.), Oxford University Press, Oxford, 1979.

7 B. Huppert, Endliche Gruppen I, Springer-Verlag, Berlin / Heidelberg / New York, 1979.

8 M. Izquierdo, On Klein surfaces and dihedral groups, Math. Scand. 76 (1995), 221-232.

9 G. A. Jones, Enumeration of homomorphisms and surface-coverings, Quarterly J. Math. Oxford (2) 46 (1995), 485-507.

10 G. A. Jones, Characters and surfaces: a survey, in The ATLAS ten years on: Proceedings, Proc. Conf. Birmingham, 1995 (eds. R. T. Curtis and R. A. Wilson), London Math. Soc. Lecture Note Ser., to appear.

11 A. D. Mednyh, Determination of the number of nonequivalent coverings over a compact Riemann surface, Dokl. Akad. Nauk SSSR 239 (1978), 269-271 (Russian); Soviet Math. Dokl. 19 (1978), 318-320 (English translation).

12 J-P. Serre, Représentations linéaires des Groupes finis, Hermann, Paris, 1967.

13 J-P. Serre, Topics in Galois Theory, Jones and Bartlett, Boston, 1992.

14 H. Zieschang, E. Vogt and H-D. Coldewey, Surfaces and Planar Discontinuous Groups, Lecture Notes in Math. 835, 1980.

DEPARTMENT OF MATHEMATICS

UNIVERSITY OF SOUTHAMPTON

SOUTHAMPTON SO17 1BJ

UNITED KINGDOM

E-mail: gaj@maths.soton.ac.uk 